\title{
A Novel Method for Contrast Enhancement with Colour Preservation
}

Raj P* and Nagpal S

Department of Computer Science and Engineering, Ppimt, Hisar 125001, India

\begin{abstract}
This paper addresses the problem of low contrast in the images. Acquiring image from low illumination source or due to some other problems the image may have low contrast which results the poor visual effect. In these type of images the different object present cannot be distinguished clearly and may not be effectively used for the desired purpose. For these images there is a need to improve the contrast for a better visual effect. This paper proposed an algorithm that can improve the contrast of the images and preserve the details of the image. This algorithm preserves the details as well as the colors of the object to a larger extent. The algorithm is tested on various images and the result of the test is given.
\end{abstract}

Keywords: Histogram; Contrast enhancement; Histogram equalization; Pixels

\section{Introduction}

Contrast refers to the distribution of gray levels to a range. For a better view of the image, it should have a good contrast of the image. Good contrast here implies the increase in the range distribution of the pixels within the allowable range. A low contrast image means the gray level of the pixels get confined to a very small range. Narrow the range become, lesser would be the contrast. The low contrast may occur due to poor illumination, less sensors used while acquiring the image, and other reasons. To use an image for the better results the image should be free from such deformities. The contrast enhancement technique deals with removing such problems from the image. The contrast enhancement technique plays a very important role in image processing field for the improvement of the quality of image. The main problem with the low contrast images is the inability to distinguish the various objects in the image. While observing the image it is very necessary to identify its constituents. The different objects in the image can be distinguished by the difference in the gray levels. The main problem with the low contrast image is that difference between gray levels of different objects in the images get reduced due to which identification of these objects become difficult. To overcome this problem the paper proposed an effective algorithm which can distribute the pixels values to large ranges by which the overall quality of image can be enhanced.

The main objective of image processing is to extract or get the information from an image. Histogram Equalization (HE) effectively extends out the most frequent intensity values, which outcome in a better distribution on the histogram. This allows for the areas of lower local contrast to gain a higher contrast without disturbing the global contrast. Image contrast enhancement is a traditional problem area in image processing. The enhancement is extensively used for medical image processing and as a preprocessing step in speech recognition, texture synthesis and many other image/video processing applications [1]. A very accepted technique for contrast enhancement of images is Histogram Equalization (HE) which could be used for all types of images. Histogram equalization performs its operation by remapping the intensity levels of the image based on the probability distribution of the input intensities [2]. The Adaptive HE [3-6] (AHE) generates the mapping for each pixel from the histogram in a nearby window. The method in [7] is to illustrate that the resulting framework can be used to produce a variety of contrast enhancement effects, of which HE is a special case by specifying alternative forms of a function i.e., cumulation function. On principle of the average intensity value as their separating point, the Brightness Preserving Bi-Histogram Equalization (BBHE) [8] and Quantized Bi-Histogram Equalization (QBHE) works. Dual Subimage Histogram Equalization (DSIHE) [9] uses the median intensity value as the separating point. Minimum Mean Brightness Error Bi-HE (MMBEBHE) [10], uses the separating point that produces the smallest Absolute Mean Brightness Error (AMBE). Recursive Mean-Separate Histogram Equalization (RMSHE) [5] is another enhancement of BBHE. The Brightness preserving dynamic histogram equalization (BPDHE) method is actually an extension to both MPHEBP and DHE. Weighting mean-separated sub-histogram equalization (WMSHE) [11] method is to perform the effective contrast enhancement of the digital image. The another method for contrast enhancement is based on is presented with a mapping function, which is a mixture of global and local transformation functions that improve both the brightness and fine details of the input image [12].

One suggested method for contrast enhancement [13] is based on the curvelet transform. The curvelet transform represents edges better than wavelets, and is therefore well-suited for multiscale edge enhancement. The results based on enhancement are better than other enhancement methods on noisy images. Contrast Enhancement of Images using Human Contrast Sensitivity is most recent advancement in image processing [14] which enhances images by controlling the local image gradient to improve the local image contrast. They attain this without segmenting the image either in the spatial (multiscale) or frequency (multi-resolution) domain. Another approach of contrast enhancement using histogram equalization with bin underflow and bin overflow [15] shows finest results. The gradient of the mapping function is controlled by applying some restrictions on the probability density function with the bin underflow (BU) and bin overflow (BO). The BUBO operation can provides the rate of enhancement from non to the full $\mathrm{HE}$ by using a single parameter. The $\mathrm{HE}$ can be utilised to execute image processing tasks such as black/white level stretch or automatic

*Corresponding author: Raj P, Department of Computer Science and Engineering Ppimt, Hisar 125001, India, Tel: 0166226 0640; E-mail: Paruraj88@gmail.com

Received January 15, 2016; Accepted February 15, 2016; Published February 18 2016

Citation: Raj P, Nagpal S (2016) A Novel Method for Contrast Enhancement with Colour Preservation. Adv Robot Autom 5: 144. doi: 10.4172/2168-9695.1000144

Copyright: (c) 2016 Raj P, et al. This is an open-access article distributed under the terms of the Creative Commons Attribution License, which permits unrestricted use, distribution, and reproduction in any medium, provided the original author and source are credited. 
brightness control as well as variable rate contrast enhancement by using enhancement rate control mechanism existing. The proposed algorithm [16] consists of two stages i.e., the first stage the poor quality image is processed by modified and enhanced sigmoid function. In second stage the output of the first stage is further processed by contrast limited adaptive histogram equalization to enhance contrast of images. A novel mask based on input value combined with the modified sigmoid formula that will be used to enhance contrast in addition to contrast limited adaptive histogram equalization. The algorithm passes over the input image which operates on its pixels one by one in spatial domain.

The section II gives the proposed algorithm and the explanation of the proposed method. The paper presented the measures to compare the quality of the images produced through histogram equalization and our proposed method. Then the results on the gray level images and coloured images are shown with the various comparison measures.

\section{Peak signal to noise ratio (PSNR)}

The experimental results have been compared using the mean square error (MSE) and peak signal-to-noise ratio (PSNR) measures that have been given below

$$
M S E=\frac{1}{\boldsymbol{m}} \sum_{x=0}^{m-1} \sum_{y=0}^{n-1}\left(A(x, y)-R(x, y)^{2}\right.
$$

where $\mathrm{A}$ and $\mathrm{R}$ are the original and the restored images having a resolution of $\mathrm{m} \times \mathrm{n}$.

$$
P S N R=10 \log _{10}\left(\frac{\max ^{2}}{M S E}\right)
$$

where max is the maximum possible pixel value of the image and its value is 255 in the case of a grayscale image.

\section{Entropy}

The entropy measure to check the contrast quality of the image. Larger the entropy more the randomness and better the contrast $[17,18]$. The formula for:

$$
H=-\sum_{i} p_{i} \log p_{i}
$$
level i.

Where $\mathrm{p}_{\mathrm{i}}$ is the probability of occurrence of pixel value with gray

By experimenting on various images we found that better contrast images show the high value of entropy. If the entropy is high then the colours of images can be preserved.

\section{Proposed Method}

In this algorithm we increase the range of the gray level as well as their randomness. If we study the histogram of a low contrast image then it can be observed that the gray levels are confined to a narrow range (Figure 1). Now to obtain the image with better contrast the values should be spread in the domain of the image intensities $[0, \mathrm{~L}]$ where $\mathrm{L}$ is the maximum value allowed in the image (for 8 bit image it is 255). Another thing that one should consider is that while increasing the range of pixels intensity in given domain, the lower values should map toward lower range i.e., towards zero and higher values toward the L. Consider the image with low contrast as X. Calculate the minimum and maximum gray level in it, then find the difference $\mathrm{d}$ between the two.

$$
\mathrm{d}=|\max (\mathrm{X})-\min (\mathrm{X})|\{\mathrm{d} \mid 1 \leq \mathrm{d} \leq 255 \text { and } \max \neq \min \}=\text { number of bins }(4)
$$

a

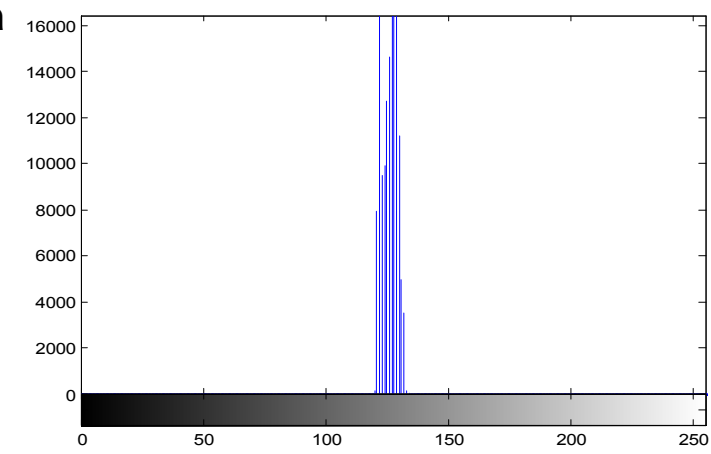

b

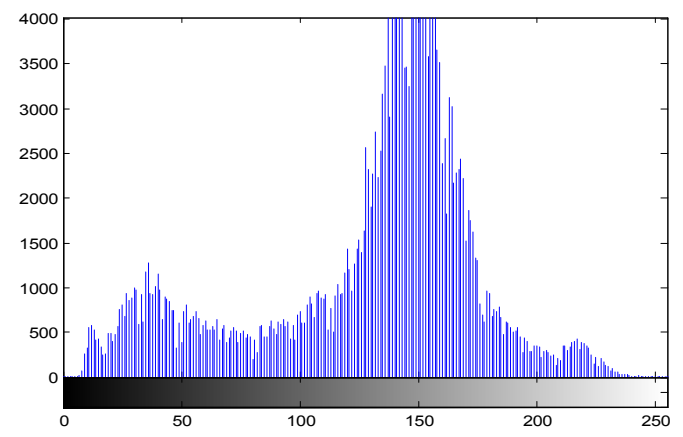

Figure 1: a) Histogram of low contrast image. b) Histogram of high contrast image.

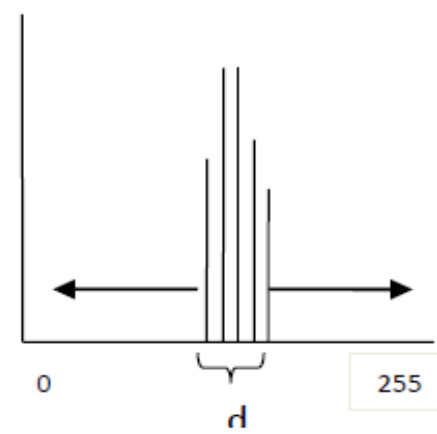

Figure 2: To show the'd' and how the pixel intensity will expand.

To expand the range of gray level we need to map them to a bigger range, for that create bins in the output image (Figure 2). The numbers of bins are equal to the value " $\mathrm{d}$ ". To step from one bin to another calculate the step function " $s$ " where,

$\mathrm{s}=\mathrm{L} / \mathrm{d}$

Every bin can contain a range of pixels value within its limit. The bin can be thought of as a containment composed of different racks and each rack can contain single pixel value. The bin size or number of racks in a bin is given by Figure 3 .

$$
\|\mathrm{b}\|=\operatorname{ceil}(\mathrm{s})
$$

The size of bin $\|\mathrm{b}\|$ signifies that every bin can have $\|\mathrm{b}\|$ such different pixel value intensity. Suppose we have $\|\mathrm{b}\|=7$ then the 


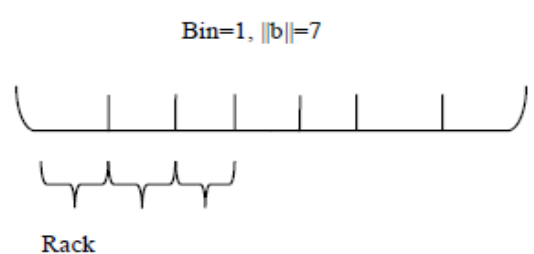

Figure 3: To show racks and bins.

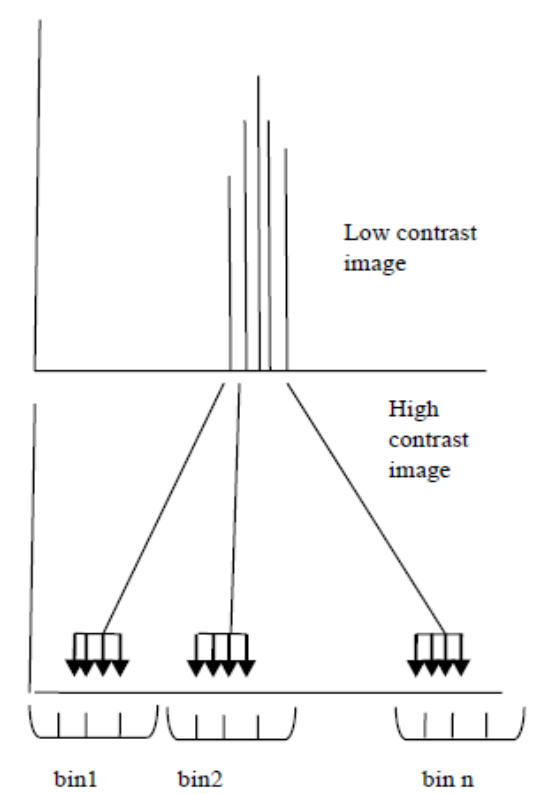

Figure 4: To show mapping of low contrast image pixels to bins of output image.

particular bin can have seven racks to put different pixel intensities. From the equations (5) and (6) this can be observed that if the contract of the image is low then the value of the $d$ would be less which increases the value of $\|b\|$. For the low contrast image the numbers of the bins would be less but each bin have large size i.e., more number of racks. In this case single pixel value from the input (low contrast) image will mapped to large number of racks in the bin. If $d \rightarrow 1$ (i.e., very small range of pixel values in input image) then $\|\mathrm{b}\| \rightarrow \mathrm{L}$ (255 for gray images) i.e., large availability of racks in the bins. If $d \rightarrow 255$ (shows that input image have good contrast) the $\|b\| \rightarrow 1$ then not much chance in mapping.

To map the single pixel value to the racks of a particular bin, randomness should be produced. This can be done by generating a random number " $\mathrm{r}$ "

$$
\mathrm{r}=\operatorname{random}(1,\|\mathrm{~b}\|)\{\mathrm{r} \mid 1 \leq \mathrm{r} \leq\|\mathrm{b}\|\}
$$

Now for every pixel in the input low contrast image, find the difference of that pixel with the minimum pixel value in the input image.

$$
\mathrm{Z}=|\mathrm{X}(\mathrm{i}, \mathrm{j})-\min (\mathrm{X})|
$$

And then map the values of the input image to new values by the given equation (Figure 4).

$$
\mathrm{Y}(\mathrm{i}, \mathrm{j})=\mathrm{z}^{*} \mathrm{~s}+\mathrm{r}
$$

The above figure shows the mapping of gray levels form input image to the resultant image. This shows how in our proposed method the same intensity value in the low contrast image maps to the different value in the output image and produce randomness and large range so that an image with better contrast can be obtained.

\section{Algorithm}

1. Input: Let $\mathrm{X}$ be the image with low contrast

2. Calculate $\mathrm{ma}(\mathrm{X})\{\mathrm{ma}(.) \leftarrow$ the pixel with maximum value

3. Calculate $\operatorname{mi}(\mathrm{X})\{\mathrm{mi}(.) \leftarrow$ the pixel with minimum value $\}$

4. $\mathrm{d} \leftarrow|\max (\mathrm{X})-\min (\mathrm{X})|\{\mathrm{d} \mid 1 \leq \mathrm{d} \leq 255 \& \max \neq \min \}$

5. $\mathrm{s}=(\mathrm{L} / \mathrm{d})$

6. $\|\mathrm{b}\| \leftarrow \operatorname{ceil}(\mathrm{s})$

$\forall \mathrm{X}(\mathrm{i}, \mathrm{j})$

$\mathrm{i} . \mathrm{z} \leftarrow|\mathrm{X}(\mathrm{i}, \mathrm{j})-\min (\mathrm{X})|$

ii. $r \leftarrow \operatorname{random}(1$, bin $)$

iii.Y $($ i.j $) \leftarrow \operatorname{round}\left(z^{\star} s\right)+r$

7. Output: $Y$ the image with improved contrast $\}$

\section{Results}

In our results, we have used ET for entropy of true image. EL stands for entropy of low contrast image. EH stands for entropy of image outcome through histogram equalization. EP stands for entropy of image through our proposed method. PSNRH is used to denote Peak to Signal Noise Ratio between true image and histogram equalized image. PSNRp is used to denote Peak to Signal Noise Ratio between true image and proposed algorithm outcome image. In our experiment the true image is that image which have proper contrast and taken in all suitable circumstances. Then we reduce the contrast of the image to very low extent to get low contrast image (Figures 5 and 6).

For the colored images our main motive was to preserve the colors of the images as likely possible as the true image. If we lose the color then the visual quality of the image got affected. Below we have shown that results through our proposed algorithm can preserve the color of very low contrast images which cannot be dine through histogram equalization (Figures 7-10) (Tables 1 and 2).
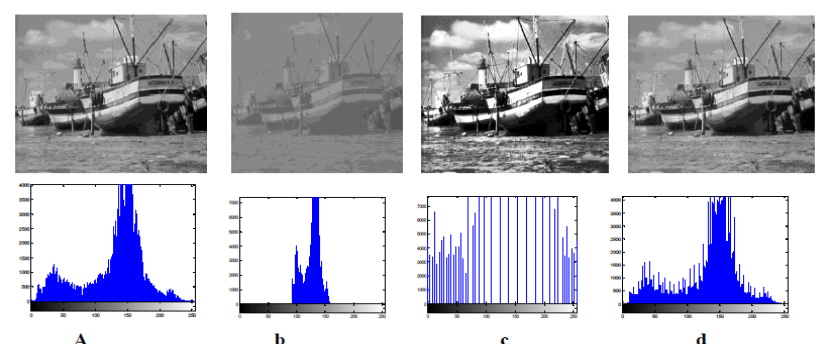

$\mathrm{ET}=7.19$
$\mathrm{EL}=5.41$

$\mathrm{EL}=5.41$
$\mathrm{EH}=5.03$

$\mathrm{EP}=7.14$

$\mathrm{PSNRH}=17.12$

Figure 5: a) True image and theirhistogram;b) Input images with low contrast and theirhistogram; c)Histogram equilization results; andd) Proposed method results. 

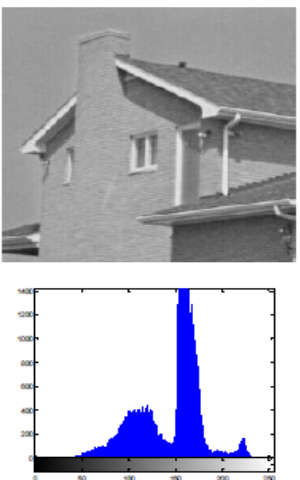

$\mathrm{ET}=6.4311$

$\mathrm{EL}=5.3430$

$\mathrm{EH}=4.8781$

$\mathrm{EP}=6.6220$

PSNRH $=14.370$

$\mathrm{PSNRP}=29.16$
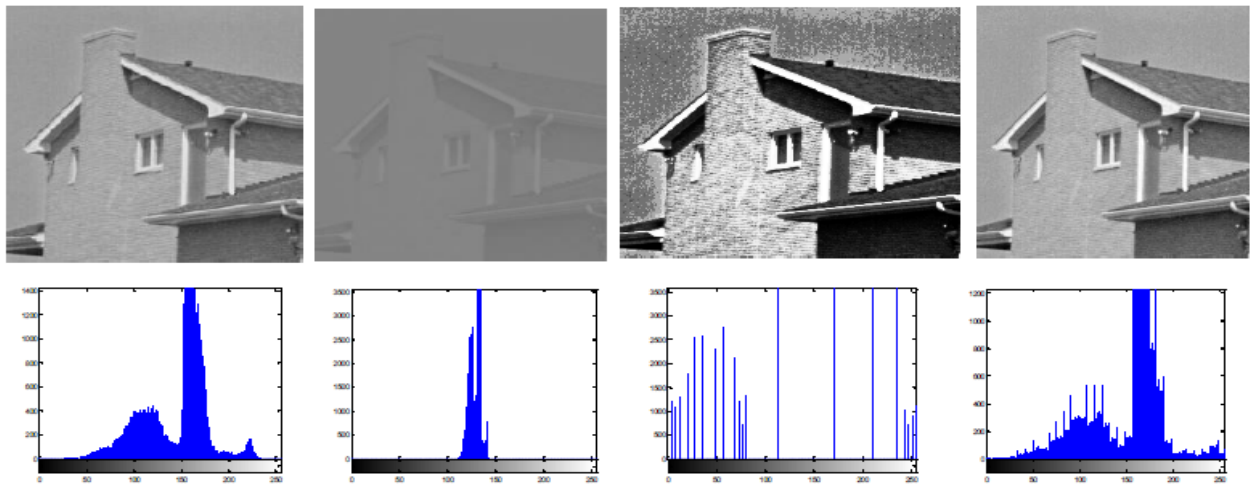

$\mathrm{ET}=6.4311$

$\mathrm{EL}=3.7303$

$\mathrm{EH}=3.6369$

$\mathrm{EP}=6.7701$

$\mathrm{PSNRH}=14.40$

PSNRP $=28.05$
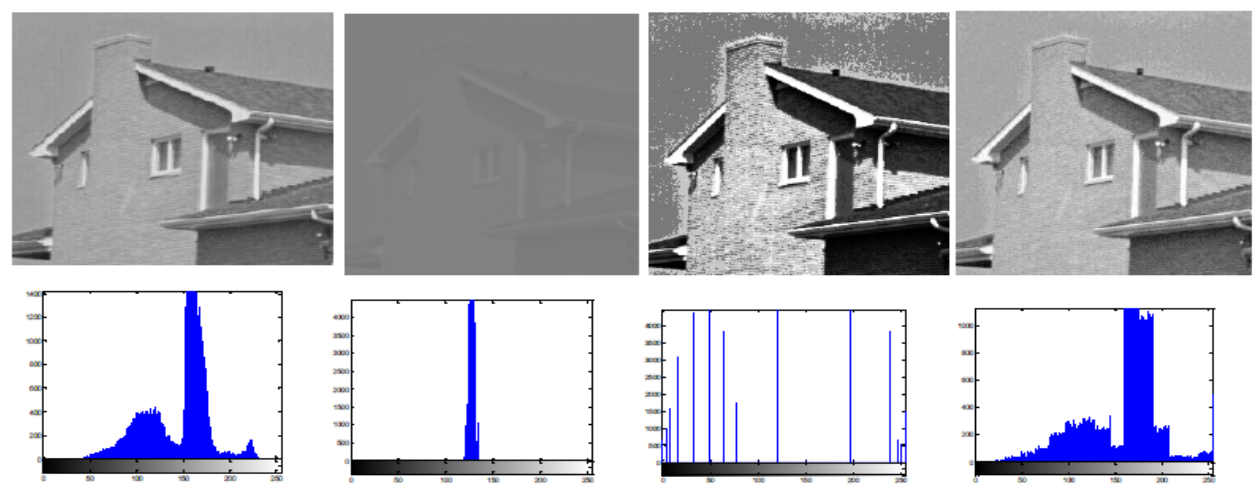

a

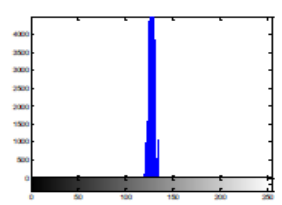

b

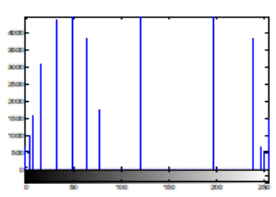

c
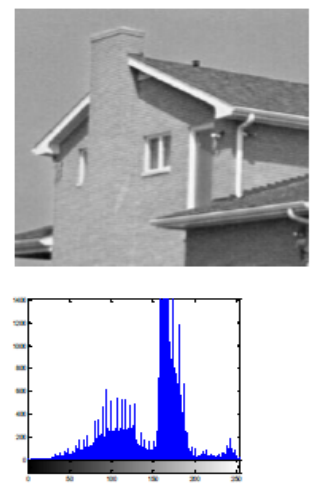

$\mathrm{ET}=6.4311$

$\mathrm{EL}=2.9479$

$\mathrm{EH}=2.9196$

$\mathrm{EP}=6.9097$

PSNRH $=14.622$

PSNRP $=23.788$

Figure 6: a) True images and their histogram; b) Input images with low contrast and their histogram; c) Histogram equilization results; and d) Proposed method results. 


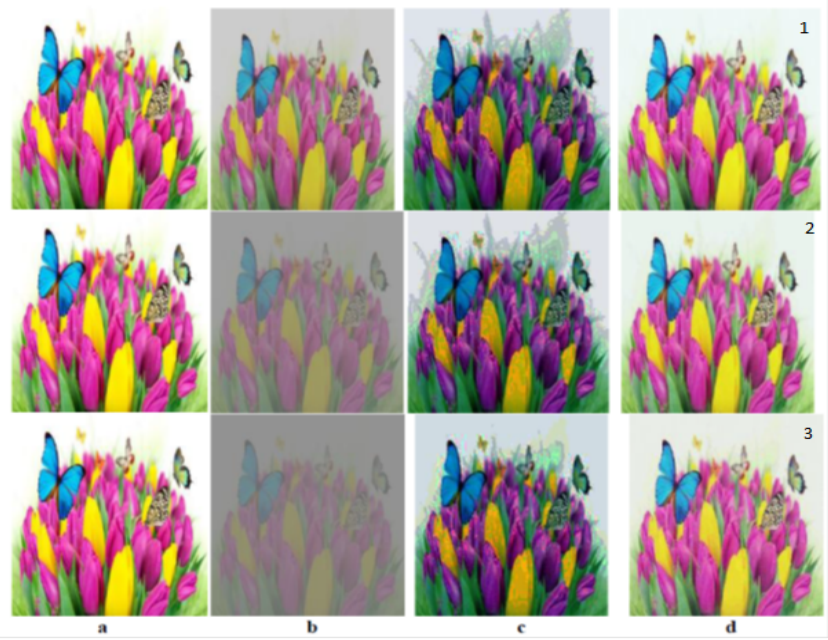

1. $\mathrm{ET}=6.8264$

$\mathrm{EL}=6.4644$
$\mathrm{EH}=5.6232$

$\mathrm{EP}=7.2783$

PSNRH $=9.158$
PSNRP $=24.2338$

2. $\mathrm{ET}=6.8264$
$\mathrm{EL}=5.3997$

$\mathrm{EL}=5.3997$
$\mathrm{EH}=5.5310$

$\mathrm{EP}=7.4111$

$\mathrm{PSNRH}=9.1690$
PSNRP $=21.9810$

3. $\mathrm{ET}=6.8264$

3. $\mathrm{ET}=6.8264$
$\mathrm{EL}=4.7482$

$\mathrm{EH}=5.2695$

$\mathrm{EP}=7.4111$
$\mathrm{PSNRH}=9.164$

PSNRP $=21.3616$

Figure 7: a) True images; b) Input images with low contrast; c) Histogram equilization results; and d) Proposed method results.
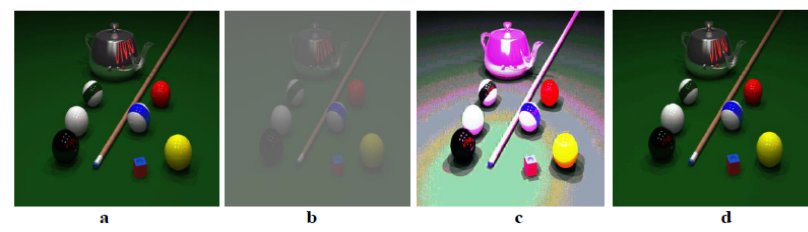

$\mathrm{ET}=5.5571$
$\mathrm{EL}=3.5363$

$\mathrm{EL}=3.5363$
$\mathrm{EH}=5.0545$

$\mathrm{EP}=5.8534$

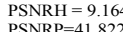

Figure 8: a) True image; b) Input images with low contrast; c) Histogram equilization results; d) Proposed method results; and e) The entropies and PSNR.
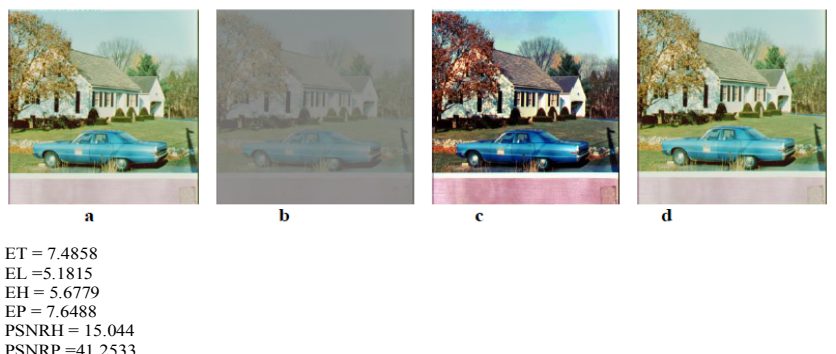

$\mathrm{EP}=7.6488$

SNRP $=41.253$

Figure 9: a) True image; b) Input images with low contrast; c) Histogram equilization results; and d) Proposed method results.
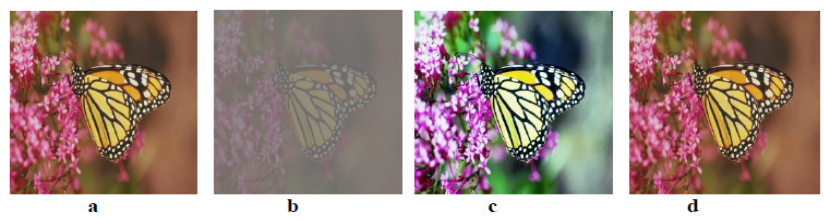

$\mathrm{ET}=7.4957$

$\mathrm{EL}=5.2292$
$\mathrm{EH}=5.5272$

$\mathrm{EH}=5.5272$
$\mathrm{EP}=7.5569$

PSNRH $=17.2180$
PSNRP $=25.9882$

Figure 10: a) True image; b) Input images with low contrast; c) Histogram equilizationresults; and d) Proposed method results.

\begin{tabular}{|c|c|c|c|c|c|c|}
\hline Fig. no. & ET & EL & EH & EP & PSNR $_{\mathbf{H}}$ & PSNR $_{\mathbf{p}}$ \\
\hline 5 & 7.19 & 5.41 & 5.03 & 7.14 & 17.12 & 39.17 \\
\hline 6 & 6.4311 & 5.3430 & 4.8781 & 6.6220 & 14.370 & 29.165 \\
\hline 6 & 6.4311 & 3.703 & 3.6369 & 6.7701 & 14.40 & 28.05 \\
\hline 6 & 6.4311 & 2.9479 & 2.9196 & 6.9097 & 14.62 & 23.788 \\
\hline
\end{tabular}

Table 1: To show the PSNR and Entropy of the gray scale images.

\begin{tabular}{|c|c|c|c|c|c|c|}
\hline Fig. no. & ET & EL & EH & EP & PSNR $_{\mathbf{H}}$ & PSNR $_{\mathbf{p}}$ \\
\hline 7 & 6.8264 & 6.44644 & 5.623 & 7.2783 & 9.158 & 24.338 \\
\hline 7 & 6.8264 & 5.3997 & 5.5310 & 7.4111 & 9.1690 & 21.968 \\
\hline 7 & 6.8264 & 4.9482 & 5.2695 & 7.4111 & 9.164 & 21.3616 \\
\hline 8 & 5.571 & 3.536 & 5.0545 & 5.853 & 9.164 & 41.822 \\
\hline 9 & 7.458 & 5.1875 & 5.677 & 7.648 & 15.044 & 41.253 \\
\hline 10 & 7.495 & 5.229 & 5.557 & 7.556 & 17.28 & 25.982 \\
\hline
\end{tabular}

Table 2: To show the PSNR and Entropy of the colour images.

\section{Conclusion}

In this paper we have proposed algorithm for the contrast enhancement of images. The results on various images are given and compared with the histogram equalization. Various measures like Entropy and PSNR is use to shoe the effectiveness of our results. In case of colored images the color quality of the images are also preserved to produce a better visual effect which is a very important factor for judging the overall quality of images. From the results it can be inferred that our proposed algorithm shows better Entropy, PSNR and visual effect than Histogram Equalization.

\section{References}

1. Pei C, Zeng C, Chang H (2004) Virtual Restoration of Ancient Chinese Paintings Using Color Contrast Enhancement and Lacuna Texture Synthesis. Computer Journal of IEEE Transactions Image Processing 13: 416-429.

2. Chen D, Ramli R (2003) Contrast Enhancement Using Recursive MeanSeparate Histogram Equalization for Scalable Brightness Preservation. Computer Journal of IEEE Transactions Consumer Electronics 49: 1301-1309.

3. Ketcham DJ, Lowe R, Weber W (1976) Seminar on image processing. RealTime Enhancement Techniques pp: 1-6.

4. Hummel R (1977) Image enhancement by histogram transformation. Comp Graph Image Process 6: 184-195.

5. Tom VT, Wolfe GJ (1982) Adaptive histogram equalization and its applications SPIE Applicat Dig Image process 359: 204-209.

6. Pizer SM, Amburn EP, Austin JD, Cromartie R, Geselowitz A, et al. (1987) Adaptive histogram equalization and its variations. Comp Vis Graph Image Process 39: 355-368.

7. Wongsritong K, Kittayaruasiriwat K, Cheevasuvit F, Dejhan K, Somboonkaew A (1998) Contrast enhancement using multipeak histogram equalization with brightness preserving. IEEE Asia-Pasific Conference on Circuit and Systems pp: 24-27. 
Citation: Raj P, Nagpal S (2016) A Novel Method for Contrast Enhancement with Colour Preservation. Adv Robot Autom 5: 144. doi:10.4172/21689695.1000140

Page 6 of 6

8. Shannon C (1948) A mathematical theory of communication. Bell Syst Tech 27: $379-423$

9. Chen SD, Ramli A (2003) Minimum mean brightness error Bi-Histogram equalization in contrast enhancement. IEEE Trans on Consumer Electronics 49: $1310-1319$

10. Wu PC, Cheng FC, Chen YK (2010) A Weighting Mean-Separated SubHistogram Equalization for Contrast Enhancement. IEEE Trans Biomedical Engineering and Computer Science.

11. Hasanul K, Abdullah A, Oksam C (2010) Brightness Preserving Image Contrast Enhancement Using Weighted Mixture of Global and Local Transformation Functions. The International Arab Journal of Information Technology 7: 403-412.

12. Jayasree PS, Raj P, Pradeep K, Rajesh S, Ghrera SP (2012) A fast novel algorithm for salt and pepper image noise cancellation using cardinal B-splines. Springer-Verlag London Limited 7: 1145-1157.
13. Starck JL, Murtagh F, Candès EL, Donoho DL (2003) Gray and Color Image Contrast Enhancement by the Curvelet Transform. IEEE Transactions on Image Processing 12: 706-717.

14. Majumder A, Irani S (2006) Contrast Enhancement of images using human contrast sensitivity. University of California, Irvine.

15. Yang S, Oh J, Park Y (2003) Contrast enhancement using histogram equalization with bin under flowand bin overflow. Digital Media R\&D Center, Korea.

16. Shyam L, Mahesh C (2014) Efficient Algorithm for Contrast Enhancement of Natural Images. The International Arab Journal of Information Technology 11: 95.

17. Shannon CE (1948) A Mathematical Theory of Communication. The Bell System Technical Journal 27: 379-423.

18. Morio J, Philippe R, Francois G, Pascale C Dubois F (2009) A Characterization of Shannon Entropy and Bhattacharyya Measure of Contrastin Polarimetric and Interferometric SAR Image, Digital Object Identifier. Proceedings of the IEEE. 